\title{
CYSTADENOMA OF THE SEMINAL VESICLE
}

\author{
ANTÔNIO O. GIL, LUCAS Y. S. YAMAKAMI, TÉRCIO GENZINI \\ Oswaldo Cruz Deutch Hospital, São Paulo, SP, Brazil
}

\begin{abstract}
Primary tumors of the seminal vesicle are extremely rare. Among them, there is a spectrum of tumors derived from both epithelium and stroma and so classified as epithelial-stromal tumors.

Herein, we report a case of a cystadenoma in a 49-year-old asymptomatic man, detected in a routine ultrasonography for liver disease follow-up. The digital rectal examination detected a large mass anterior to rectum and posterior to bladder. Computed tomography scan and magnetic resonance imaging showed a normal prostate and a $9.0 \mathrm{~cm}$ cystic tumor, replacing the left seminal vesicle. The gross appearance and microscopic aspect was compatible with cystadenoma of seminal vesicle. Patient's postoperative recovery was uneventful. He is currently alive, 3 years after the diagnosis, with no signs of recurrence.
\end{abstract}

Key words: seminal vesicle; neoplasms, glandular and epithelial; cystadenoma Int Braz J Urol. 2003; 29: 434-6

\section{INTRODUCTION}

Primary tumors of the seminal vesicle are extremely rare. Among them, there is a spectrum of tumors derived from both epithelium and stroma (1$3)$. Herein, we present a patient with an epithelialstromal tumor in this unusual location.

\section{CASE REPORT}

A 49-year-old asymptomatic man with chronic hepatitis-C was at surveillance after 1 year treatment with interferon, when a routine ultrasonography demonstrated a pelvic mass. Physical examination was unremarkable except for digital rectal examination, which detected a large mass anterior to rectum and posterior to bladder, with a normal prostate. Computed tomography (CT) scan and magnetic resonance imaging showed a normal prostate and a $9 \mathrm{~cm}$ well-defined expansive tumor, predominantly cystic, with septations, replacing the left semi- nal vesicle (Figure-1). No upper tract abnormalities were seen.

The patient was explored through a midline abdominal incision and a complex mass posterior to bladder was identified. A direct approach to the retrovesical space was chosen, and the tumor was totally dissected from attachments to the bladder anteriorly, rectum posteriorly and surrounding tissues, and fully removed, without any incision in bladder or rectum.

The tumor measured $7.0 \times 5.0 \times 4.5 \mathrm{~cm}$. It was covered by a gray capsule and there was a remanescent of the seminal vesicle with $3.5 \times 1.2 \mathrm{~cm}$ dimensions. Through the external surface, multiple cavities were seen, fulfilled with a clear liquid. Histologic examination showed dilated cysts, covered by a single layer of cuboidal epithelium, without atypia (Figure-2). Microscopic aspect was compatible with cystadenoma of the seminal vesicle.

The patient postoperative recovery was uneventful. One year after surgery, a CT scan showed 
no signs of recurrence. He is currently alive and well, 3 years after the diagnosis.

\section{DISCUSSION}

We reported here a rare case of a cystadenoma of the seminal vesicle. Usually, these benign tumors have only minor complaints, such as nonspecific lower abdominal pain or urinary frequency (1-3). Diagnosis should be considered in the presence of a mass located superior to prostate and posterior to bladder, protruding the rectal wall $(1,3)$.

Macroscopically, they are multilocular tumors. Microscopically, they have epithelial and stromal components combined. The first delimits cystic areas, lined by a single layer of cuboidal or columnar epithelium (3). The second is formed by fibrous connective tissue, which may resemble fibroblasts or smooth muscle cells (3).

Differential diagnosis is made with expansive injuries of retrovesical space such as carcinomas and cystic tumors. Immunohistochemical analysis using prostate specific antigen and prostatic acid phosphatase may be done to exclude prostatic origin (1-3). In the present case, macroscopic and microscopic appearance was typi-

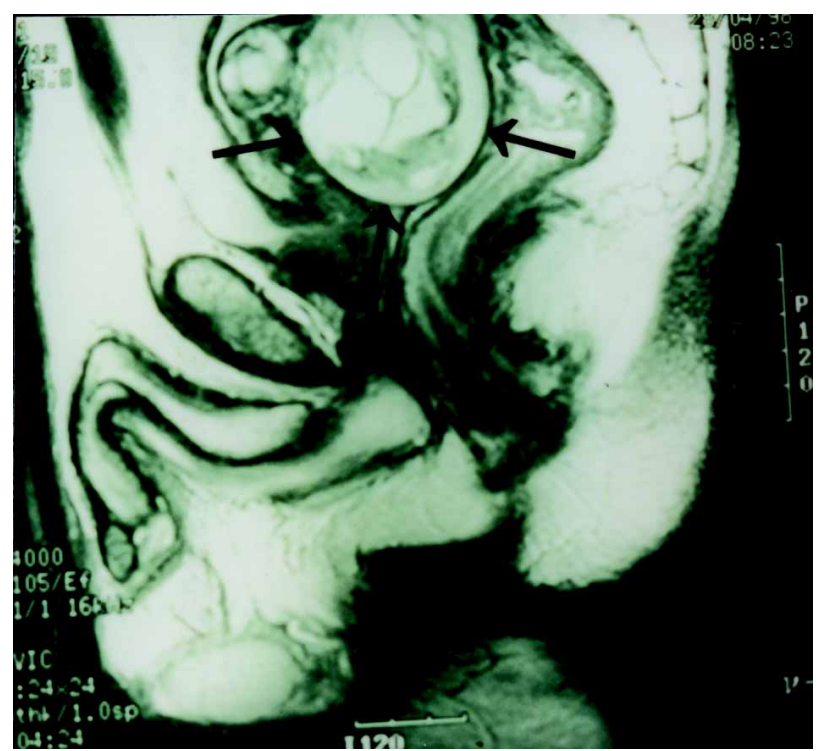

Figure 1 - Pelvic magnetic resonance imaging demonstrating a multilocular tumor (arrows) between bladder and rectum.

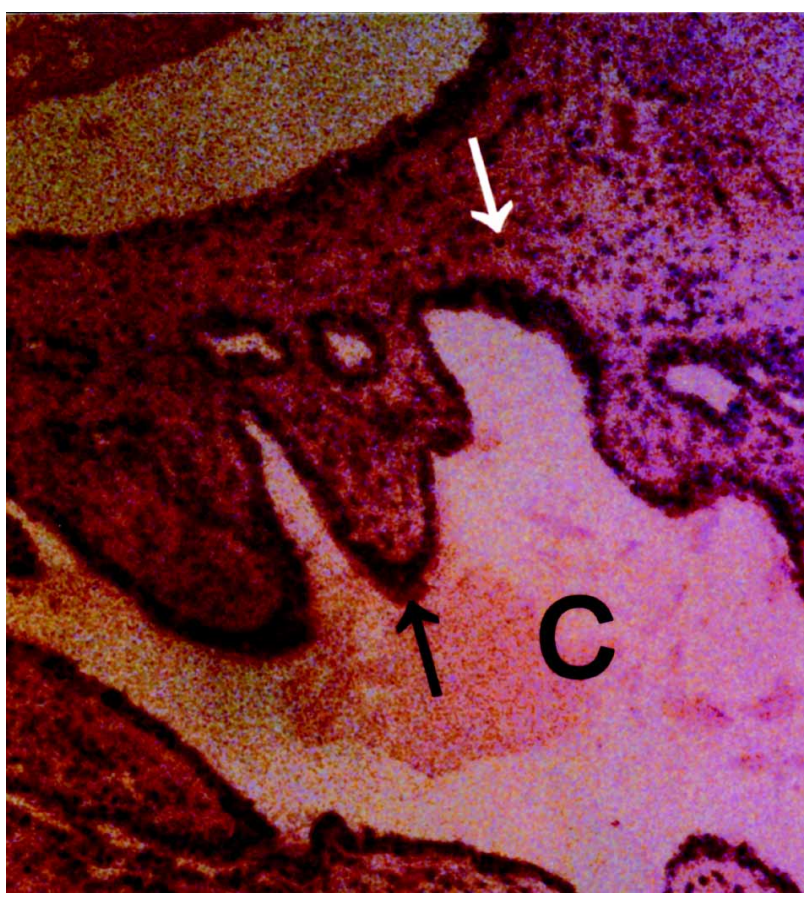

Figure 2 - Histologic examination showing dilated cysts $(C)$ covered by a single layer of cuboid epithelium (black arrow) and filled by amorphous eosinophilic material. The stroma is composed of spindle-shaped cells, without significant atypia (white arrow). There are no mitotic figures or solid epithelial proliferation (HE, X100).

cal of a primary benign epithelial-stromal tumor of the seminal vesicle and so, more properly called cystadenoma (3).

There is not large experience on management of these neoplasms. Marsupialization and aspiration are not indicated due to their multilocular organization. Because fine needle aspiration biopsies may be inconclusive $(1,2)$, an exploratory laparotomy is usually needed and, in this case, it is logical to remove the whole tumor and involved seminal vesicle. When surgical treatment is successful, prognosis is invariably good, without recurrence.

\section{REFERENCES}

1. Mazzucchelli L, Studer UE, Zimmermann A: Cystadenoma of the seminal vesicle: case report and literature review. J Urol. 1992; 147: 1621-4. 
2. Baschinsky DY, Niemann TH, Maximo CB, Bahnson RR: Seminal vesicle cytadenoma: a case report and literature review. Urology 1998; 51: 840-5.
3. Santos LD, Wong CSKC, Killingsworth M: Cystadenoma of the seminal vesicle: report of a case with ultrastructural findings. Pathology 2001; 33: 399402.

Received: April 25, 2003 Accepted after revision: June 27, 2003

\section{Correspondence address:}

Dr. Antônio Otero Gil

Rua Cotoxó, $611 / 10^{\circ}$ andar / 105

São Paulo, SP, 05021-000, Brazil

Fax: + $55113873-5782$

E-mail: doctorgil@uol.com.br 